\title{
POINTWISE MEASURABLE FUNCTIONS
}

\author{
HERMANN RENDER and LOTHAR ROGGE
}

\begin{abstract}
We introduce the new concept of pointwise measurability. It is shown in this paper that a measurable function is measurable at each point and that for a large class of topological spaces the converse also holds. Moreover it can be seen that a function which is continuous at a point is Borel-measurable at this point too. Furthermore the set of measurability points is considered. If the range space is a $\sigma$-compact metric space, then this set is a $G_{\delta}$-set; if the range space is only a Polish space this is in general not true any longer.
\end{abstract}

\section{Introduction}

Continuity of a function $f: X \rightarrow Y$ between two topological spaces $(X, \tau)$ and $(Y, \rho)$ is usually defined at a given point $x \in X$. It is an amazing fact that Borel-measurability of a function $f: X \rightarrow Y$ is defined in a global way by requiring that the preimage of a Borel set is a Borel set and it seems to be impossible to give an adequate definition of pointwise measurability.

We introduce in this paper a notion of measurability of a function $f: X \rightarrow$ $Y$ at a given point $x \in X$, which satisfies the following conditions for many topological spaces:

(i) if $f$ is continuous at $x$, then it is measurable at $x$;

(ii) if $f$ is measurable, then it is measurable at each point $x \in X$;

(iii) if $f$ is measurable at each point $x \in X$, then it is measurable.

More precisely our definition of pointwise measurability fulfills (i) if the range space $Y$ is Hausdorff, fulfills (ii) for all topological spaces $X$ and $Y$, and (iii) for a large class of topological spaces.

It is a well known fact that the set of continuity points of a function $f$ : $X \rightarrow Y$, namely the set of all $x \in X$ such that $f$ is continuous at $x$, is a $G_{\delta}$-set if $Y$ is a separable metric space. In the context of pointwise measurability it is natural to consider the set

$$
M_{f}:=\{x \in X: f \text { is measurable at } x\},
$$

Received January 24, 2003. 
which we call the set of measurability points of $f$. If $Y$ is a $\sigma$-compact metric space, then $M_{f}$ is a $G_{\delta}$-set, a result which is no longer true for separable metric spaces.

\section{The main results}

Our basic assumptions throughout the paper are the following: $(X, \tau)$ and $(Y, \rho)$ are topological spaces. Furthermore $\mathscr{A}$ is a $\sigma$-algebra with $\mathscr{A} \supset \tau$ and $\sigma(\rho)$ is the $\sigma$-field generated by $\rho$, i.e. the Borel- $\sigma$-field of $Y$.

A function $f: X \rightarrow Y$ is called measurable if it is $\mathscr{A}, \sigma(\rho)$-measurable. If furthermore $\mathscr{A}=\sigma(\tau)$, then we call $f$ Borel-measurable.

Our approach of pointwise measurability will be formulated in nonstandard terms, which was the original motivation of this work. However, we also give a standard characterization of pointwise measurability which is less elegant than the nonstandard characterization. For nonstandard notions see [3] or [6].

The $\mathscr{A}$-monad of $y \in{ }^{*} X$ and relation $\approx_{\mathscr{A}}$ are defined by

$$
m_{\mathscr{A}}(y)=\cap_{y \in A \in \mathscr{A}}{ }^{*} A \quad \text { and } \quad z \approx_{\mathscr{A}} y \Longleftrightarrow z \in m_{\mathscr{A}}(y) .
$$

The following result is due to Ross [10], see also [4].

Let $\mathscr{A}_{1}$ and $\mathscr{A}_{2}$ be $\sigma$-algebras over $X$ and $Y$. Then $f: X \rightarrow Y$ is $\mathscr{A}_{1}, \mathscr{A}_{2-}$ measurable iff

$$
z \approx_{\mathscr{A}_{1}} y \Rightarrow{ }^{*} f(z) \approx_{\mathscr{A}_{2}}{ }^{*} f(y) .
$$

Loeb [7] showed a result which was generalized in [5]. Let $f: X \rightarrow \mathrm{R}$ be a bounded function. Then $f$ is measurable iff

$$
z \approx_{\mathscr{A}} y \Rightarrow s t^{*} f(z)=s t^{*} f(y) .
$$

Here $s t(a)$ denotes the standard part of an element $a \in{ }^{*} \mathrm{R}$. Now we come to our definition of measurability at a point $x$.

Definition. Let $f: X \rightarrow Y$. Then $f$ is called measurable at $x \in X$ if for all $u \in Y$ the following condition holds

$$
z \approx_{\mathscr{A}} y \wedge y \approx_{\tau} x \wedge{ }^{*} f(y) \approx_{\rho} u \Rightarrow^{*} f(z) \approx_{\rho} u .
$$

If $\mathscr{A}=\sigma(\tau)$ we call $f$ Borel-measurable at $x$.

Here $y \approx_{\tau} x$ is defined by $y \in m(x):=\cap_{x \in U \in \tau}{ }^{*} U$.

The following theorem gives standard characterizations of measurability of a function $f$ at a point $x$.

Theorem 2.1. Let $f: X \rightarrow Y$ be a function and $x \in X$. Then the following statements are equivalent: 
(i) $f$ is measurable at $x$.

(ii) For each compact set $K \subset Y$, for each open neighborhood $V$ of $K$ there exist an open neighborhood $W$ of $K$, an open neighborhood $U$ of $x$ and a set $A \in \mathscr{A}$ such that

$$
f^{-1}(W) \cap U \subset A \subset f^{-1}(V) .
$$

(iii) For each $u \in Y$, for each open neighborhood $V$ of $u$ there exist an open neighborhood $W$ of $u$, an open neighborhood $U$ of $x$ and a set $A \in \mathscr{A}$ such that

$$
f^{-1}(W) \cap U \subset A \subset f^{-1}(V) .
$$

If $Y$ is a prehausdorff space - i.e. two points of $Y$ which cannot be separated by neighborhoods have the same system of neighborhoods - then it can be seen by 2.1(iii) that a function which is continuous at $x$ is Borel-measurable at $x$. A counterexample in $\$ 3$ shows that this is in general not true for an arbitrary topological space $Y$.

From 2.1(iii) also follows, that a measurable function is measurable at each point. Hence (ii) of the introduction holds. The following simple example shows that the converse (i.e. (iii) of the introduction) is not true in general.

ExAmple 2.2. Let $X$ be the unit interval $[0,1]$ with the euclidean topology and let $Y=[0,1]$ be endowed with the discrete topology. Then it is easy to see that the function $f$, defined by $f(x)=x$, is Borel-measurable at each $x \in X$ (use 2.1(iii) with $W=\{u\}$ ), but clearly $f$ is not Borel-measurable.

This example shows that we need a certain kind of countability condition for the space $Y$. In the following we give some sufficient criteria such that pointwise measurable functions are measurable. For this we recall that a topological space $(X, \tau)$ is cosmic (see [8]) if the space is regular and if there exists a countable system $\left(F_{k}\right)_{k \in \mathrm{N}}$ of sets such that for all $x \in X$, for all open neighborhoods $V$ of $x$ there exists $k \in \mathrm{N}$ such that $x \in F_{k} \subset V$. By Proposition 10.2 in [8] a space is cosmic if and only if it is a continuous image of a separable metric space. Recall that a space $X$ is Lindelöf if each open covering of the space has a countable subcovering. A space is hereditarily Lindelöf if each subspace is Lindelöf. It is known that a cosmic space is hereditarily Lindelöf.

THEOREM 2.3. Let $(X, \tau)$ be hereditarily Lindelöf and $(Y, \rho)$ be a cosmic space. If a function $f: X \rightarrow Y$ is measurable at each point $x \in X$ then $f$ is measurable.

The following example shows that we also need a certain kind of countability condition for the space $X$ in the theorems 2.3 and 2.5 . 
EXAmPLe 2.4. There exists a locally compact Hausdorff space $X$ and a function $f: X \rightarrow \mathrm{R}$ which is Borel-measurable at each point, but which is not Borel-measurable.

If we require that the image space is a $\sigma$-compact metric space (a property stronger than cosmic) we can relax the condition on $X$ to be Lindelöf:

THEOREM 2.5. Let $(X, \tau)$ be a Lindelöf space and $(Y, \rho)$ be a $\sigma$-compact metric space. If a function $f: X \rightarrow Y$ is measurable at each $x \in X$ then $f$ is measurable.

So far we have assumed that the image space is the image of a separable metric space, hence it is of small cardinality. If we assume that $Y$ is a $\sigma$ compact space the cardinality of $Y$ can be big and we have to distinguish between Borel and Baire sets. Recall that a set $U$ is functionally open if there exists a continuous real-valued function $f$ such that $U=f^{-1}(0,1)$. A map $f: X \rightarrow Y$ is called weakly measurable if $f^{-1}(U) \in \mathscr{A}$ for each functionally open set $U$.

THEOREM 2.6. Let $(X, \tau)$ be a Lindelöf space and $(Y, \rho)$ be $\sigma$-compact completely regular space. If a function $f: X \rightarrow Y$ is measurable at each $x \in X$ then $f$ is weakly measurable.

In the following we give results about the set

$$
M_{f}(\mathscr{A})=\{x \in X: f \text { is } \mathscr{A} \text {-measurable at } x\} .
$$

A point $x$ is a condensation point of a topological space if every neighborhood of $x$ is uncountable. Then we have

THEOREM 2.7. Let $(Y, \rho)$ be a $\sigma$-compact metric space and $f: X \rightarrow Y$ be a function. Then $M_{f}(\mathscr{A})$ is a $G_{\delta}$-set. If moreover $(X, \tau)$ is a hereditarily Lindelöf, Hausdorff space then every point of $X \backslash M_{f}(\mathscr{A})$ is a condensation point in the relative topology of $X \backslash M_{f}(\mathscr{A})$.

To prove that $M_{f}(\mathscr{A})$ is a $G_{\delta}$-set it is not sufficient to assume that $(Y, \rho)$ is a Polish space:

EXAMPLE 2.8. Let $X=[0,1]$ be endowed with the euclidean topology and $Y=\mathrm{R}^{\mathrm{N}}$ with the product topology. Let $\mathscr{B}$ be the Borel- $\sigma$-algebra on $X$. Then there exists a function $f: X \rightarrow \mathrm{R}^{\mathrm{N}}$ such that $M_{f}(\mathscr{B})$ is not a $G_{\delta}$-set.

If we weaken the assumption on $(Y, \rho)$ further we obtain

Example 2.9. Let $X=[0,1]$ be endowed with the euclidean toplogy and $Y=[0,1]$ with the discrete topology. Then there exists a function $f$ : $X \times X \rightarrow Y \times\{0,1\}$ such that $M_{f}(\mathscr{B})$ is not a Borel-set. 
If we assume only that $Y$ is a complete uniform instead of complete metric space we have the following example

ExAmple 2.10. Let $X=[0,1]$ be endowed with the euclidean topology and let $\emptyset \neq A \subset[0,1]$. Then there exists a function $f: X \rightarrow \mathbf{R}^{A}$ such that $M_{f}(\mathscr{B})=A$.

Now consider the case $Y=\mathrm{R}$. Let $(X, \delta)$ be a fixed Polish space. We know by Theorem 2.7 that $M_{f}:=M_{f}(\mathscr{B})$ is a $G_{\delta}$-set, and moreover that a point of $X \backslash M_{f}$ is a condensation point in the relative topology of $X \backslash M_{f}$. The following examples show that essentially each such subset is the set of non-measurability points of a function $f: X \rightarrow \mathrm{R}$.

Example 2.11. Let $(X, \delta)$ be a Polish space such that every point is a condensation point, then there exist a function $f: X \rightarrow \mathrm{R}$ such that $M_{f}(\mathscr{B})=$ $\emptyset$.

ExAmple 2.12. Let $(X, \delta)$ be a Polish space and $X_{0}=\cup_{n=1}^{\infty} C_{n} \subset X$ where $C_{n}$ is closed and each point of $C_{n}$ is a condensation point of $C_{n}$. Then there exists a function $f: X \rightarrow \mathrm{R}$ such that $X \backslash X_{0}=M_{f}(\mathscr{B})$.

The following example shows that a function $f: X \rightarrow \mathrm{R}$ can be measurable at a point $x_{0}$, whereas $f^{+}$and $f \wedge n$ are not measurable at $x_{0}$.

ExAmPLE 2.13. Let $X=[0,1]$ be endowed with the euclidean topology. Then there exists a function $f: X \rightarrow \mathrm{R}$ wich is Borel-measurable at $x_{0} \in X$ whereas $f^{+}$and $f \wedge n(n \in \mathrm{N})$ are not Borel-measurable at $x_{0}$.

Let $M_{f}=M_{f}(\mathscr{A})$. In the situation of Theorem 2.7 an arbitrary function $f$ can be decomposed in a measurable function $f \mid M_{f}$ and a function $f \mid X \backslash M_{f}$ which is measurable at no point.

TheORem 2.14. Let $(X, \tau)$ be a hereditarily Lindelöf, Hausdorff space, $Y$ a $\sigma$-compact metric space and $f: X \rightarrow Y$ be a function. Then $f_{1}:=f \mid M_{f}$ is an $\mathscr{A} \cap M_{f}$-measurable function, hence $M_{f_{1}}=M_{f}$, and $f_{2}:=f \mid X \backslash M_{f}$ has the property that $M_{f_{2}}$ is empty.

\section{The proofs of 2 and some further results}

Proof of Theorem 2.1. Let $m_{\rho}(K):=\cap_{K \subset O \in \rho}{ }^{*} O$ and

$$
\left.m_{\mathscr{A}}(C)=\cap{ }^{*} A: C \subset{ }^{*} A \wedge A \in \mathscr{A}\right\} .
$$

(i) $\Rightarrow$ (ii) Let $K \subset Y$ be a compact set, then

$$
m_{\rho}(K)=\cup_{u \in K} m(u) .
$$


We show

$$
z \approx_{\mathscr{A}} y \wedge y \approx_{\tau} x \wedge^{*} f(y) \in m_{\rho}(K) \Rightarrow{ }^{*} f(z) \in m_{\rho}(K) .
$$

As $* f(y) \in m_{\rho}(K)$, by (1) there exists $u \in K$ with $* f(y) \approx_{\rho} u$. As $f$ is pointwise measurable at $x$, we have ${ }^{*} f(z) \approx_{\rho} u$ and hence ${ }^{*} f(z) \in m_{\rho}(K)$.

Choose $W \in{ }^{*} \rho$ with ${ }^{*} K \subset W \subset m_{\rho}(K)$ and let $U \in{ }^{*} \tau$ be choosen with ${ }^{*} x \in U \subset m(x)$. We show

$$
m_{\mathscr{A}}\left({ }^{*} f^{-1}(W) \cap U\right) \subset{ }^{*} f^{-1}\left({ }^{*} V\right) .
$$

To prove (3) let $z \in m_{\mathscr{A}}\left(f^{-1}(W) \cap U\right)$ be given. As ${ }^{*} f^{-1}(W) \cap U$ is intern, there exists $y \in{ }^{*} f^{-1}(W) \cap U$ with $z \approx_{\mathscr{A}} y$. Hence $y \in U \subset m(x)$, whence $y \approx_{\tau} x$. Since ${ }^{*} f(y) \in W \subset m_{\rho}(K)$ we obtain ${ }^{*} f(z) \in m_{\rho}(K) \subset{ }^{*} V$ by (2). Hence (3) is proven.

A saturation argument shows that there exists $A \in *^{*} \mathscr{A}$ with ${ }^{*} f^{-1}(W) \cap U \subset$ $A \subset{ }^{*} f^{-1}(V)$. Transfer finishes the proof of (i) $\Rightarrow$ (ii).

The proof of (ii) $\Rightarrow$ (iii) is trivial.

(iii) $\Rightarrow$ (i) Let $z \approx_{\mathscr{A}} y \approx_{\tau} x$ and ${ }^{*} f(y) \approx_{\rho} u \in Y$. Let furthermore $V \in \rho$ with $u \in V$ be choosen. We have to show

$$
{ }^{*} f(z) \in{ }^{*} V .
$$

Choose $W, A, U$ according to (iii). Then

$$
{ }^{*} f^{-1}\left({ }^{*} W\right) \cap{ }^{*} U \subset{ }^{*} A \subset{ }^{*} f^{-1}\left({ }^{*} V\right) .
$$

As ${ }^{*} f(y) \approx_{\rho} u$ and $u \in W \in \tau$ we have ${ }^{*} f(y) \in{ }^{*} W$. Moreover $y \in{ }^{*} U$ and hence $y \in{ }^{*} f^{-1}\left({ }^{*} W\right) \cap{ }^{*} U$. By (5) we have $y \in{ }^{*} A$ with $A \in \mathscr{A}$. As $z \approx_{\mathscr{A}} y$ we obtain $z \in{ }^{*} A$ and hence ${ }^{*} f(z) \in{ }^{*} V$ by (5), i.e. (4) holds.

Proof of Theorem 2.3. Let $\left(F_{k}\right)_{k \in \mathrm{N}}$ be a system of $(Y, \rho)$ as in the definition of a cosmic space. Let $V$ be an open subset of $Y$. Define (4)

$$
X_{k}:=\left\{x \in X:(\exists U \in \tau)(\exists A \in \mathscr{A}): x \in f^{-1}\left(F_{k}\right) \cap U \subset A \subset f^{-1}(V)\right\} .
$$

For each $x \in X_{k}$ choose $U_{x}(k) \in \tau$ and $A_{x}(k) \in \mathscr{A}$ such that $x \in f^{-1}\left(F_{k}\right) \cap$ $U_{x}(k) \subset A_{x}(k) \subset f^{-1}(V)$. Then $\left(U_{x}(k)\right)_{x \in X_{k}}$ is an open covering of $X_{k}$. Since $X$ is hereditarily Lindelöf there exists a countable subcovering, say $\left(U_{x_{i}}(k)\right)_{i \in \mathrm{N}}$, of $X_{k}$. Then $X_{k} \subset \cup_{i=1}^{\infty} U_{x_{i}}(k)$. Since $X_{k} \subset f^{-1}\left(F_{k}\right)$ we obtain

$$
X_{k} \subset \cup_{i=1}^{\infty}\left(U_{x_{i}}(k) \cap f^{-1}\left(F_{k}\right)\right) \subset \cup_{i=1}^{\infty} A_{x_{i}}(k) \subset f^{-1}(V) .
$$

By the pointwise measurability of $f$ at $x$ it is easy to see that $f^{-1}(V)$ is the union of the sets $X_{k}$ (use 2.1.3). Hence $f^{-1}(V)=\cup_{k, i=1}^{\infty} A_{x_{i}}(k) \in \mathscr{A}$. 


\section{Example 2.4}

Let $\Omega$ be the first uncountable ordinal and $X$ be the set of all ordinals less than $\Omega$. Then $X$ with the order topology is a locally compact completely normal Hausdorff-space (see [10], p.p. 68-69). Since $X$ has cardinality $\aleph_{1}$, there does not exist according to Ulam a probability measure $\mu$ on the power set of $X$ with $\mu\{x\}=0$ for all $x \in X$. However according to Halmos (see [2], Example 10, p. 231) there exists a probability measure $\mu$ on the Borel- $\sigma$-field $\mathscr{B}(X)$ with $\mu\{x\}=0$ for all $x \in X$. Hence there exists a set $B \in X$ with $B \notin \mathscr{B}(X)$ and $f=1_{B}$ is not Borel-measurable. However $f$ is Borel-measurable at each $x \in X$; use 2.1(iii) with the countable open set $U:=[0, x+1[$.

Before we can prove Theorem 2.5 we need the following result:

THEOREM 3.1. Let $(X, \tau)$ be a Lindelöf space. If a function $f: X \rightarrow Y$ is measurable at each $x \in X$ then for each compact set $K \subset Y$ and for each open neighborhood $V$ of $K$ there exists a set $A \in \mathscr{A}$ such that

$$
f^{-1}(K) \subset A \subset f^{-1}(V) .
$$

In particular:

$$
f^{-1}(K) \in \mathscr{A} \text { for each compact } G_{\delta}-\text { set } K \text { in } Y .
$$

Proof. Let us prove at first formula (1): Let $K$ be compact and $V$ be an open neighborhood of $K$. For each $x \in X$ there exist by 2.1(iii) an open neighborhood $W_{x}$ of $K$, an open neighborhood $U_{x}$ of $x$ and a set $A_{x} \in \mathscr{A}$ such that

$$
f^{-1}\left(W_{x}\right) \cap U_{x} \subset A_{x} \subset f^{-1}(V) .
$$

Then $\left(U_{x}\right)_{x \in X}$ is an open covering of $X$ and by the Lindelöf property there exists a countable subcover, say $\left(U_{x_{i}}\right)_{i \in \mathrm{N}}$. Since $X=\cup_{i=1}^{\infty} U_{x_{i}}$ we obtain

$$
f^{-1}(K) \subset \bigcup_{i=1}^{\infty}\left(U_{x_{i}} \cap f^{-1}(K)\right) \text {. }
$$

Since $f^{-1}(K) \subset f^{-1}\left(W_{x}\right)$ formula (2) shows that

$$
f^{-1}(K) \subset \bigcup_{i=1}^{\infty} A_{x_{i}} \subset f^{-1}(V) .
$$

Hence (1) is proven. To prove $f^{-1}(K) \in \mathscr{A}$ for a compact $G_{\delta}$-set $K$ note that there exist open sets $V_{n}$ such that $K=\cap_{n=1}^{\infty} V_{n}$. By (1) there exists $A_{n} \in \mathscr{A}$ 
such that $f^{-1}(K) \subset A_{n} \subset f^{-1}\left(V_{n}\right)$. Hence $f^{-1}(K)=\cap_{n=1}^{\infty} A_{n} \in \mathscr{A}$. This proves (2).

Proof of Theorem 2.5. The Borel $\sigma$-algebra of $Y$ is generated by the compact $G_{\delta}$-sets. Now apply (2) of Theorem 3.1.

Proof of Theorem 2.6. The Baire $\sigma$-algebra of $Y$ defined as the $\sigma$-algebra generated by the functionally open sets is in this case generated by the compact $G_{\delta}$-sets. Now apply (2) of Theorem 3.1.

Definition. Let $T \subset X$ and $x \in X$. Then $x$ is called a measurability point of $T$ if $1_{T}$ is measurable at $x$. If $\mathscr{A}=\sigma(\tau)$, then $x$ is called a Borelmeasurability point of $T$.

We obtain from 2.1 the following simple criterion for measurability points. Let $\bar{T}:=X \backslash T$.

CRITERION FOR A MEASURABILITY POINT 3.2. Let $T \subset X$ and $x \in X$. Then the following statements are equivalent

(i) $x$ is a measurability point of $T$;

(ii) $y \approx_{\tau} x \Rightarrow m_{\mathscr{A}}(y) \subset{ }^{*} T$ or $m_{\mathscr{A}}(y) \subset \overline{{ }^{*} T}$;

(iii) $\exists O \in \mathscr{T}$ with $x \in O$ and $O \cap T \in \mathscr{A}$.

Proof. (i) $\Rightarrow$ (iii) According to 2.1(iii) the measurability of $f=1_{T}$ at $x$ is equivalent to the existence of open sets $U_{1}, U_{2}$ and sets $A_{1}, A_{2} \in \mathscr{A}$ with $x \in U_{1} \cap U_{2}$ and

$$
T \cap U_{1} \subset A_{1} \subset T, \bar{T} \cap U_{2} \subset A_{2} \subset \bar{T} .
$$

Then $O:=U_{1}$ fulfills $T \cap O=A_{1} \cap O \in \mathscr{A}$.

(iii) $\Rightarrow$ (i) Put $U_{i}:=O, A_{1}:=O \cap T, A_{2}=O \backslash O \cap T \in \mathscr{A}$, then (1) and hence (i) holds.

(i) $\Leftrightarrow$ (ii) Let $f=1_{T}$, then ${ }^{*} f=1{ }^{*} T$ and hence $x$ is a measurability point of $f \Longleftrightarrow\left[\left(z \approx_{\mathscr{A}} y \approx_{\tau} x\right) \Rightarrow\left(y \in{ }^{*} T \Rightarrow z \in^{*} T\right) \wedge\left(y \notin^{*} T \Rightarrow z \notin^{*} T\right)\right]$. This shows the equivalence of (i) and (ii).

From 3.2 it follows that a measurability point of $T$ is a measurability point of $\bar{T}$; furthermore the points of measurability of a set are open.

If $(Y, \rho)$ is a metric space with metric $\rho$ we write for $y, z \in{ }^{*} Y$

$$
y \approx z \Longleftrightarrow{ }^{*} \rho(y, z) \approx 0 .
$$

If $(Y, \mathscr{V})$ is a uniform space we write for $y, z \in{ }^{*} Y$

$$
y \approx z \Longleftrightarrow(\forall V \in \mathscr{V})(y, z) \in{ }^{*} V .
$$


In 3.3 we give a characterization of the measurability of a function $f: X \rightarrow Y$ if $f(X)$ is a relatively compact subset of the metric space $Y$. Let in the following $d$ be a metric generating the topology $\rho$ of $Y$.

Theorem 3.3. Let $(X, \tau)$ be a topological and $(Y, \rho)$ be a metric space. Let $f: X \rightarrow Y$ be a function such that $f(X)$ is relatively compact. Then the following statements are equivalent for a fixed $x \in X$ :

(i) $f$ is measurable at $x$;

(ii) $z \approx_{\mathscr{A}} y \wedge y \approx_{\tau} x \Rightarrow^{*} f(z) \approx^{*} f(y)$;

(iii) For each $\varepsilon>0$ there exists a neighborhood $U$ of $x$ and a partition $A_{1}, \ldots, A_{n} \in \mathscr{A}$ such that for all $i \in\{1, \ldots, n\}$

$$
y, z \in U \cap A_{i} \Rightarrow d(f(y), f(z))<\varepsilon .
$$

Proof. The equivalence of (i) and (ii) follows from the fact that each point of ${ }^{*} f\left({ }^{*} X\right)$ is a nearstandard point. The equivalence of (ii) and (iii) follows from the more general result 3.4 with $\mathscr{F}=\{Y\}$.

THEOREM 3.4. Let $(X, \tau)$ be a topological and $(Y, \mathscr{V})$ be a uniform space. Let $f: X \rightarrow Y$ and $\mathscr{F}$ be a filter over $Y$. Then the following statements are equivalent for a fixed $x \in X$ :

(i) $z \approx_{\mathscr{A}} y \wedge y \approx_{\tau} x \wedge{ }^{*} f(y) \in m_{\mathscr{F}} \Rightarrow{ }^{*} f(z) \approx{ }^{*} f(y)$;

(ii) For each $V \in \mathscr{V}$ there exists a neighborhood $U$ of $x$ and a partition $A_{1}, \ldots, A_{n} \in \mathscr{A}$ and $F \in \mathscr{F}$ such that for all $i=1, \ldots, n$

$$
y, z \in U \cap A_{i} \wedge f(y) \in F \Rightarrow(f(y), f(z)) \in V .
$$

Proof. Let $\mathscr{U}_{\mathscr{A}}$ be the filter generated by the sets $S_{A}:=A \times A \cup(\bar{A} \times \bar{A})$ with $A \in \mathscr{A}$. Then $\mathscr{U}_{\mathscr{A}}$ induces the relation $\approx_{\mathscr{A}}$, i.e.

$$
m_{U_{\mathscr{A}}}=\left\{(y, z) \in{ }^{*} X \times{ }^{*} X: y \approx_{\mathscr{A}} z\right\} .
$$

For (i) $\Rightarrow$ (ii) let $V \in \mathscr{V}$. Assumption (i) implies

(1) $(y, z) \in m_{\mathscr{U}_{A}} \wedge y, z \in m(x) \wedge{ }^{*} f(y) \in m(\mathscr{F}) \Rightarrow\left({ }^{*} f(z),{ }^{*} f(y)\right) \in{ }^{*} V$.

Choose $U \in{ }^{*} \tau$ with $U \subset m(x)$ and $F \in{ }^{* \mathscr{F}}$ with $F \subset m(\mathscr{F})$. By saturation there exists a hyperfinite set $\mathscr{C}$ such that $\left\{{ }^{*} A: A \in \mathscr{A}\right\} \subset \mathscr{C} \subset{ }^{*} \mathscr{A}$. We can require that $\mathscr{C}$ is an algebra. Then

$$
\cap_{A \in \mathscr{C}}(A \times A \cup(\bar{A} \times \bar{A})) \subset m_{\mathscr{U}_{\mathscr{A}}} .
$$


Denote by $\mathscr{Z}(\mathscr{A})$ the set of all finite partitions $A_{1}, \ldots, A_{n} \in \mathscr{A}$. As $\mathscr{C}$ is a hyperfinite internal algebra, there exists $\mathrm{A} \in{ }^{*} \mathscr{Z}(\mathscr{A})$, where $A:\{1, \ldots, n\} \rightarrow$ $* \mathscr{A}$ is an internal partition with

$$
\cap_{i=1}^{n} \mathrm{~A}(i) \times \mathrm{A}(i) \subset m_{\mathscr{U}_{\mathscr{A}}}
$$

(use (2)). Hence by (1) the following statement is true, where $\tau_{x}:=\{O \in \tau$ : $x \in O\}, \mathscr{D}(\mathrm{A})$ is the set on which $\mathrm{A}$ is defined and $A_{i}:=\mathrm{A}(i)$,

$$
\begin{array}{r}
\left(\exists U \in{ }^{*} \tau_{x}\right)\left(\exists \mathrm{A} \in{ }^{*} \mathscr{Z}(\mathscr{A})\right)\left(\exists F \in{ }^{*} \mathscr{F}\right)(\forall i \in \mathscr{D}(\mathrm{A}))\left(\forall y, z \in U \cap A_{i}\right)\left({ }^{*} f(y)\right. \\
\left.\in F \Rightarrow\left({ }^{*} f(y),{ }^{*} f(z)\right) \in{ }^{*} V\right) .
\end{array}
$$

By transfer the statement follows.

For (ii) $\Rightarrow$ (i) let $z \approx_{\mathscr{A}} y, y \approx_{\tau} x$ and ${ }^{*} f(y) \in m(\mathscr{F})$ be given. Choose $V \in \mathscr{V}$. Then there exists $U \in \tau_{x}$, a partition $A_{1}, \ldots, A_{n} \in \mathscr{A}$ and a set $F \in \mathscr{F}$ such that the following statement is true by transfer

$$
(\forall i \in\{1, \ldots, n\})\left(\left(\forall y, z \in{ }^{*} U \cap{ }^{*} A_{i}\right)\left({ }^{*} f(y) \in{ }^{*} F \Rightarrow\left({ }^{*} f(y),{ }^{*} f(z)\right) \in{ }^{*} V\right) .\right.
$$

Since $z \approx_{\mathscr{A}} y$ and $y \approx_{\tau} x$ we know that $y, z \in{ }^{*} U$. As $A_{1}, \ldots, A_{n} \in \mathscr{A}$ is a partition we have $y \in{ }^{*} A_{i}$ for some $i$ and then $z \in{ }^{*} A_{i}$ by $z \approx_{\mathscr{A}} y$. As ${ }^{*} f(y) \in{ }^{*} F$ by ${ }^{*} f(y) \in m(\mathscr{F})$ it follows $\left({ }^{*} f(y),{ }^{*} f(z)\right) \in{ }^{*} V$ by (3). As $V \in \mathscr{V}$ is arbitrary we obtain ${ }^{*} f(z) \approx{ }^{*} f(y)$.

Proof of Theorem 2.7. We show at first that $M_{f}$ is a $G_{\delta}$-set. Let $X$ be the union of compact sets $\emptyset \neq K_{k}, k \in \mathrm{N}$, and let $\mathscr{F}_{k}$ be the filter of all neighborhoods of $K_{k}$. Then

$$
\cup_{k \in \mathrm{N}} m_{\mathscr{F}_{k}}=n s\left({ }^{*} Y\right) .
$$

Let $G(n, k)=\left\{x \in X:\left(\exists U \in \tau_{x}\right)(\exists \mathrm{A} \in \mathscr{Z}(\mathscr{A}))\left(\exists F \in \mathscr{F}_{k}\right)(\forall i \in\right.$ $\mathscr{D}(\mathrm{A}))\left(\forall y, z \in U \cap A_{i}\right)(f(y) \in F \Rightarrow d(f(y), f(z))<1 / n\}$. Then $G(n, k)$ is open by definition, and it is sufficient to show that

$$
M_{f}=\cap_{n, k \in \mathrm{N}} G(n, k) .
$$

By Theorem 3.4 applied to $\mathscr{F}=\mathscr{F}_{k}$ we obtain $x \in \cap_{n \in \mathrm{N}} G(n, k)$ iff $z \approx_{\mathscr{A}}$ $y \wedge y \approx_{\tau} x \wedge{ }^{*} f(y) \in m_{\mathscr{F}_{k}} \Rightarrow{ }^{*} f(z) \approx{ }^{*} f(y)$.

Hence (2) follows from (1) using the definition of pointwise measurability.

Let now $X$ be a hereditarily Lindelöf, Hausdorff space. We claim

(3) Each point in $X \backslash M_{f}$ is a condensation point in its relative topology.

Recall that a point $z$ in a topological space $Z$ is a condensation point if each neighborhood of $z$ is an uncountable set. To prove (3) assume indirectly that 
there exists $x \in Z:=X \backslash M_{f}$ which has a countable open neighborhood $U_{0}$ in the subspace topology of $Z$. Write $U_{0}=U \cap Z$ where $U$ is an open subset of $X$. We shall prove that $x \in M_{f}$ and this contradiction shows (3). Let now

$$
z \approx_{\mathscr{A}} y \wedge y \approx_{\tau} x \wedge^{*} f(y) \approx_{\rho} u
$$

We have to prove

$$
{ }^{*} f(z) \approx_{\rho} u
$$

Assume at first that $y \in{ }^{*} Z$. Let now $V$ be an open neighborhood of $u$. Then

$$
A:=f^{-1}(V) \cap U \cap Z \in \mathscr{A},
$$

since $A$ is countable and hence by the Hausdorff property of $X$ is contained in $\mathscr{A}$. Since $y \approx_{\tau} x,{ }^{*} f(y) \approx_{\rho} u$ (see (4)) and $y \in{ }^{*} Z$ we conclude that $y \in{ }^{*} A$ by (6). Now $z \approx_{\mathscr{A}} y$ implies $z \in{ }^{*} A$ and hence ${ }^{*} f(z) \in{ }^{*} V$. Since this holds for all neighborhoods $V$ of $u$ we have ${ }^{*} f(z) \approx_{\rho} u$, i.e. (5) holds.

In the second case we know that $y \in{ }^{*} M_{f}$. As $M_{f} \in \mathscr{A}$ we obtain $z \in{ }^{*} M_{f}$ by (4). By definition of $M_{f}$ the function $f$ is measurable at each point $x_{0} \in M_{f}$. Trivially $g:=f \mid M_{f}$ is $\mathscr{A} \cap M_{f}$-measurable at each point $x_{0} \in M_{f}$. Now $M_{f}$ is a Lindelöf space with $\mathscr{A} \cap M_{f} \supset \tau \cap M_{f}$. Hence by theorem $2.5 \mathrm{~g}$ is measurable in the usual sense. Apply now Theorem 3.5 to $X:=M_{f}$ and $f:=g$. As $z, y \in{ }^{*} M_{f}$ and $z \approx_{\mathscr{A} \cap M_{f}} y,{ }^{*} f(y) \in p n s_{\rho}{ }^{*} Y$ by (4), we obtain ${ }^{*} f(z) \approx_{\rho}{ }^{*} f(y) \approx_{\rho} u$ and hence ${ }^{*} f(z) \approx_{\rho} u$, i.e. (5) holds.

Theorem 3.5. Let $(Y, \mathscr{V})$ be a uniform space and let $f: X \rightarrow Y$. If $f$ is weakly measurable then $f$ satisfies

$$
z \approx_{\mathscr{A}} y \wedge^{*} f(y) \in \operatorname{pns\mathscr {V}}\left({ }^{*} Y\right) \Rightarrow{ }^{*} f(z) \approx{ }^{*} f(y)
$$

Proof. Let $V \in \mathscr{V}$ be arbitrary and $V_{1} \in \mathscr{V}$ with $V_{1} \circ V_{1} \subset V$. By a well known theorem there exists a pseudometric $d$ on $Y$ and $\varepsilon>0$ such that $W:=$ $\{(u, v): d(u, v) \leq \varepsilon\} \subset V_{1}$ and $W \in \mathscr{V}$. Then $W[\mathscr{V}]=\{u: d(u, v) \leq \varepsilon\}$ is a functionally closed set for each $v \in Y$. As ${ }^{*} f(y) \in p n s \mathcal{V}\left({ }^{*} Y\right)$ there exists $v \in Y$ with ${ }^{*} f(y) \in{ }^{*} W[v]$. Since $A:=f^{-1}(W[v]) \in \mathscr{A}$ by assumption and $z \approx_{\mathscr{A}} y$ we have $z \in{ }^{*} A$. Hence $\left({ }^{*} f(z),{ }^{*} v\right) \in{ }^{*} W$ and $\left({ }^{*} v,{ }^{*} f(y)\right) \in{ }^{*} W$. Hence $\left({ }^{*} f(y),{ }^{*} f(z)\right) \in{ }^{*} V$. It follows that ${ }^{*} f(z) \approx_{\mathscr{V}}{ }^{*} f(y)$. 
Example 2.11

Let $(X, \delta)$ be a Polish space such that every point is a condensation point. Then there exists a set $T \subset X$ without any Borel-measurability point.

Proof. There exists a Bernstein set $T \subset X$, i.e. a set such that for each uncountable closed set $C$ we have $C \cap T \neq \emptyset$ and $C \cap \bar{T} \neq \emptyset$ (see for instance the proof of Theorem 3.10 pp. 101-102 of [1]). Now we obtain

$$
\begin{aligned}
& B \in \mathscr{B}(X) \wedge B \subset T \Rightarrow B \text { is countable; } \\
& B \in \mathscr{B}(X) \wedge B \subset \bar{T} \Rightarrow B \text { is countable. }
\end{aligned}
$$

It suffices to prove (1): If $B$ is uncountable, then there exists an uncountable compact set $K \subset B \subset T$ (see for instance Theorem 2.8, p. 12 of [9]), contradicting $K \cap \bar{T} \neq \emptyset$. Now we shall show

$$
T \text { has no Borel-measurability point. }
$$

Assume indirectly that $x$ is a Borel-measurability point of $T$. Then there exists an open neighborhood $O$ of $x$ such that $O \cap T \in \mathscr{B}(X)$ and hence $O \cap \bar{T} \in$ $\mathscr{B}(X)$. Therefore $O \cap T$ and $O \cap \bar{T}$ are countable by (1) and (2). Hence $O$ is countable, contradicting the fact that $x$ is a condensation point of $X$.

\section{Example 2.12}

Apply Example 2.11 to each closed subset $C_{n}$ of $X$ separately. Hence for each $C_{n}$ there exists a Bernstein subset $T_{n}$. Then the Borel-measurability points of $T_{n}$ in $C_{n}$ are void and the Borel-measurability points of $1_{T_{n}}$ in $X$ is therefore $X \backslash C_{n}$. Define now

$$
f:=\sum_{n=1}^{\infty} 3^{-n} 1_{T_{n}} .
$$

Then $M_{f}(\mathscr{B})=\cap_{n=1}^{\infty} M_{1_{T_{n}}} \equiv \cap_{n=1}^{\infty} \overline{C_{n}}=\overline{X_{0}}$.

Example 2.8 and 2.10

Let $T \subset[0,1]$ be a set without any Borel-measurability points (for existence see Example 2.11). Let $x_{0} \in[0,1]$ be given and consider the function $f_{x_{0}}\left(x_{0}\right)=$ 0 , and $f_{x_{0}}(x)=\left(x-x_{0}\right)^{-2}\left(1_{T}-1_{\bar{T}}\right)$.

Let now $\emptyset \neq A \subset[0,1]$. Consider the function $f=\left(f_{x}\right)_{x \in A}:[0,1] \rightarrow$ $\mathrm{R}^{A}$. We shall show that

$$
M_{f}(\mathscr{B})=A .
$$

This proves 2.10. If we choose for $A$ the set of all rational points of $[0,1]$ we obtain 2.8 as $\mathbf{R}^{A}$ is isometric to $\mathbf{R}^{\mathrm{N}}$. 
To (1): Let $a \in A, z \approx_{\mathscr{B}} y \approx_{\tau} a$. If ${ }^{*} f(y) \approx u \in \mathbf{R}^{A}$, then ${ }^{*} f(y) \approx$ $u_{a} \in \mathbf{R}$ and hence $y=a$, i.e. $f$ is Borel-measurable at $a$. Let now $x_{1} \notin A$, choose points (use 3.2) $y_{1} \approx_{\tau} x_{1}, y_{2} \approx_{\mathscr{B}} y_{1}$ and $y_{1} \in{ }^{*} T, y_{2} \in{ }^{*} T$. Then ${ }^{*} f\left(y_{1}\right) \approx\left(\left(x_{1}-a\right)^{-2}\right)_{a \in A}$, however ${ }^{*} f\left(y_{2}\right) \approx\left(-\left(x_{1}-a\right)^{-2}\right)_{a \in A}$. Hence $f$ is not Borel-measurable at $x_{1}$.

\section{Example 2.9}

Take a subset $T$ of $X:=[0,1]$ such that

$$
T \cap O \notin \mathscr{B}([0,1]) \text { for all non-void subsets } O \text { of }[0,1] .
$$

Define $f: X \times X \rightarrow Y \times\{0,1\}$ by

$$
f(x, y):= \begin{cases}(x, 0) & \text { for }(x, y) \in T \times \bar{T} \\ (x, 1) & \text { for }(x, y) \in T \times T \\ (x, 0) & \text { for }(x, y) \in \bar{T} \times[1 / 2,1], \\ (x, 1) & \text { for }(x, y) \in \bar{T} \times[0,1 / 2[.\end{cases}
$$

Then $f$ is a surjective map. It is sufficient to show that

$$
M_{f}(\mathscr{B})=\bar{T} \times[0,1] .
$$

Since $Y \times\{0,1\}$ is discrete, $f$ is measurable at $(x, y)$ (see 2.1(iii)) iff for each $u \in Y \times\{0,1\}$ there exists a neighborhood $U$ of $(x, y)$ such that $f^{-1}(u) \cap$ $U \in \mathscr{A}$, where $\mathscr{A}$ is the Borel- $\sigma$-field of $[0,1] \times[0,1]$. Let $u=(z, 0)$ or $(z, 1)$. If $z \neq x$ we can choose $U$ such that $f^{-1}(u) \cap U=\emptyset$ and hence $f^{-1}(u) \cap U \in \mathscr{A}$. Therefore we may assume that $z=x$. If $(x, y) \in \bar{T} \times[0,1]$, then $f^{-1}(u)$ is equal to $\{x\} \times[1 / 2,1]$ and $\{x\} \times[0,1 / 2$ [ respectively; it follows that $(x, y) \in M_{f}(\mathscr{B})$. Let now $(x, y) \in T \times[0,1]$. Then $f^{-1}(u)$ is equal to $\{x\} \times \bar{T}$ and $\{x\} \times T$ respectively. Using (1) it follows that $(x, y) \notin M_{f}(\mathscr{B})$.

Example 3.6. Let $X=[0,1]$ be endowed with the euclidean topology. Then there exists a topological space $Y$ such that for $x_{0} \in X$ there exists a function $f: X \rightarrow Y$ which is continuous at $x_{0}$ without being Borel-measurable at $x_{0}$.

Proof. Let $T$ be a subset of $[0,1]$ such that $\bar{T} \cap O \notin \mathscr{B}(X)$ for each non-void open set $O$. We may assume that $x_{0} \in T$. Consider $Y=\{0,1\}$ with $\tau=\{\emptyset,\{0\}, Y\}$ and define $f: X \rightarrow\{0,1\}$ by $f=1_{T}$. Then $f$ is continuous at $x_{0}$ as $f\left(x_{0}\right)=1$. If $f$ would be measurable at $x_{0}$, then according to 2.1(iii) for $V:=\{0\}$ and hence $W=\{0\}$ there would exist a Borel-set $B$ and an open neighborhood $O$ of $x$ with $\bar{T} \cap O \subset B \subset \bar{T}$. Hence $\bar{T} \cap O \in \mathscr{B}$, contradicting the choice of $T$. 


\section{Example 2.13}

Consider the function $f_{x_{0}}$ of Example 2.10. Then $f_{x_{0}}$ is measurable at $x_{0}$. However $f_{x_{0}}^{+}=\left(x-x_{0}\right)^{-2} 1_{T}$ is not measurable at $x_{0}$. To this aim choose $z \in{ }^{*} T, y \in{ }^{*} T$ with $z \approx_{\mathscr{B}} y \approx_{\tau} x_{0}$. Then ${ }^{*} f_{x_{0}}^{+}(y)=0$ and ${ }^{*} f_{x_{0}}^{+}(z) \not \approx 0$. Hence $f_{x_{0}}^{+}$is not measurable at $x_{0}$. It follows similarly that $f_{x_{0}} \wedge n$ is not measurable at $x_{0}$.

ProOF OF 2.14. As $f_{1}=f \mid M_{f}$ is a pointwise measurable function, $f_{1}$ is an $\mathscr{A} \cap M_{f}$ measurable function by 2.5 . Hence it suffices to show that $M_{f_{2}}=\emptyset$. Assume that there exists $x \in M_{f_{2}} \subset X \backslash M_{f}$. Then there exists $u \in Y$ and $z, y \in{ }^{*} X$ with $z \approx_{\mathscr{A}} y$ and $y \approx_{\tau} x$ such that ${ }^{*} f(y) \approx_{\rho} u$ but ${ }^{*} f(z) \notin m(u)$. In the first case assume that $y \in{ }^{*} M_{f}$. Since $M_{f} \in \mathscr{A}$ by 2.7 it follows that $z \in{ }^{*} M_{f}$. Hence $z \underset{\mathscr{A} \cap M_{f}}{\approx} y$ and therefore by 3.5 as $f \mid M_{f}$ is measurable, ${ }^{*} f(z) \approx{ }^{*} f(y)$ contradicting that ${ }^{*} f(y) \approx \approx_{\rho} u$ and ${ }^{*} f(z) \notin m(u)$. Hence $y \notin{ }^{*} M_{f}$ and therefore $z \notin^{*} M_{f}$. We obtain $z \underset{\mathscr{A} \cap \bar{M}_{f}}{\approx} y \approx_{\tau} x$ and ${ }^{*} f_{2}(y) \approx_{\rho} u$ but $* f_{2}(z) \notin m(u)$, contradicting $x \in M_{f_{2}}$.

We would like to thank the referee for improving the presentation.

\section{REFERENCES}

1. Elstrodt, Jürgen, Maß- und Integrationstheorie, Springer Verlag, Berlin and Heidelberg, 1996.

2. Halmos, Paul, Measure Theory, D. van Nostrand Company, Inc., Princeton and New York, 1964, MR 11, $504 \mathrm{~d}$.

3. Hurd, A. E., and Loeb, P. A., Introduction to Nonstandard Real Analysis, London Math. Soc. Stud. Texts 10 (1988), MR 87 d: 03184.

4. Landers, Dieter, and Rogge, Lothar, Pointwise measurability in nonstandard models, Math. Scand. 67 (1990), 255-258, MR 92 e: 28014.

5. Landers, Dieter, and Rogge, Lothar, Nonstandard criteria for Borel-measurability, Math. Scand. 86 (2000), 244-248, MR 20019: 28006.

6. Landers, Dieter, and Rogge, Lothar, Nichtstandard Analysis, Springer Verlag, Heidelberg and New York, 1994, MR 95 i : 03140.

7. Loeb, Peter, A nonstandard representation of measurable spaces, $L_{\infty}$ and $L_{\infty}^{*}$, in Contributions to Nonstandard Analysis (W. A. J. Luxemburg and A. Robinson, eds.), North-Holland Publ., Amsterdam, 1972, 65-80, MR 57 \# 16517.

8. Michael, M., $\mathscr{X}_{0}$-spaces, J. Math. Mech. 15 (1966), 983-1002, MR 34 \# 3551.

9. Parthasarathy, K. R., Probability measures on metric spaces, Academic Press, New-York and London, 1967, MR 37 \# 2271.

10. Ross, D. A., Measurable transformations in saturated models of analysis, Ph.D. thesis University of Wisconsin, 1983. 
11. Steen, Lynn, and Seebach Jr., Arthur, Counterexamples in Topology, Springer Verlag, New York and Berlin, 1978, MR 96 k: 54001.

\author{
INSTITUT FÜR MATHEMATIK DER \\ GERHARD-MERCATOR-UNIVERSITÄT GHS DUISBURG \\ LOTHARSTR. 65 \\ D-47048 DUISBURG \\ GERMANY \\ E-mail: render@math.uni-duisburg.de,rogge@math.uni-duisburg.de
}

